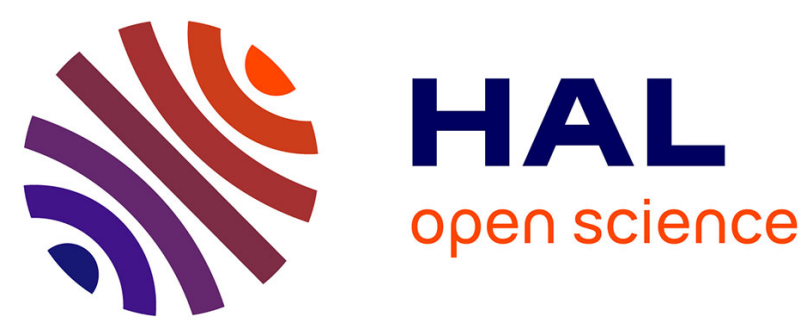

\title{
Beta spectrometry with metallic magnetic calorimeters
}

\author{
Martin Loidl, Matias Rodrigues, Cindy Le Bret, Xavier Mougeot
}

\section{To cite this version:}

Martin Loidl, Matias Rodrigues, Cindy Le Bret, Xavier Mougeot. Beta spectrometry with metallic magnetic calorimeters. Applied Radiation and Isotopes, 2014, Proceedings of the 19th International Conference on Radionuclide Metrology and its Applications 17-21 June 2013, Antwerp, Belgium, 87, pp.302 - 305. 10.1016/j.apradiso.2013.11.024 . hal-01894701

\section{HAL Id: hal-01894701 \\ https://hal.science/hal-01894701}

Submitted on 13 Feb 2020

HAL is a multi-disciplinary open access archive for the deposit and dissemination of scientific research documents, whether they are published or not. The documents may come from teaching and research institutions in France or abroad, or from public or private research centers.
L'archive ouverte pluridisciplinaire HAL, est destinée au dépôt et à la diffusion de documents scientifiques de niveau recherche, publiés ou non, émanant des établissements d'enseignement et de recherche français ou étrangers, des laboratoires publics ou privés. 


\title{
Beta spectrometry with metallic magnetic calorimeters
}

\author{
M. Loidl ${ }^{1}$, M. Rodrigues, C. Le-Bret, X. Mougeot \\ LNE - Laboratoire National Henri Becquerel, CEA Saclay, DRT/LIST/DM2I, 91191 Gif-sur- \\ Yvette, France
}

\begin{abstract}
Metallic magnetic calorimeters are a specific type of cryogenic detectors that have been shown to enable precise measurement of the shape of low energy beta spectra. The aim of their use at LNHB is the determination of the shape factors of beta spectra. The beta source is enclosed in the detector absorber, allowing for very high detection efficiency. It has turned out that the type of source is of crucial importance for the correctness of the measured spectrum. Spectra of ${ }^{63} \mathrm{Ni}$ measured with several sources prepared by drying a $\mathrm{NiCl}_{2}$ solution differ from one another and from theory, whereas spectra measured with electroplated sources are reproducible and agree with theory. With these latter measurements we could confirm the atomic exchange effect down to very low energy $(200 \mathrm{eV})$.
\end{abstract}

Keywords: Beta spectrometry, cryogenic detectors, metallic magnetic calorimeters, ionizing radiation metrology, nuclear data.

\section{Introduction}

Since several years the LNHB is developing metallic magnetic calorimeters (MMCs) for beta spectrometry. The aim is the precise determination of shape factors. Metallic magnetic calorimeters (Enss et al., 1999; Fleischmann et al., 2005) are a specific type of cryogenic ${ }^{1}$ Corresponding author. LNE - Laboratoire National Henri Becquerel, CEA Saclay, DRT/LIST/DM2I, 91191 Gif-sur-Yvette, France.
Tel.: + 331 69084277, Fax: + 331 69082619, E-mail: martin.loidl@cea.fr 
detectors that may be adapted to soft and hard X-ray, alpha, beta, and optical photon detection. For beta spectrometry, the source is enclosed in the detector absorber. Since the solid angle is $4 \pi \mathrm{sr}$ and the absorber size can be chosen as a function of the endpoint energy of the beta emitter, a detection efficiency of practically $100 \%$ can be realized for the whole spectrum, starting from a very low energy threshold typical for these detectors.

Several years ago, the spectrum of ${ }^{241} \mathrm{Pu}$ was measured with a MMC at LNHB (Loidl et al., 2010). A discrepancy between the experimental and a theoretical spectrum raised the question whether this discrepancy reflects a distortion of the experimental spectrum whose reason would need to be revealed, or is due to an insufficiency of the calculation of the theoretical spectrum: ${ }^{241} \mathrm{Pu}$ decays via a first forbidden, non-unique transition, and the spectra of forbidden, nonunique transitions are not straightforward to calculate. Consequently, the decision was taken to validate the potential of MMCs for beta spectrometry by measuring the spectrum of an allowed transition that can be calculated more reliably for comparison with the experimental spectrum. The pure beta emitter ${ }^{63} \mathrm{Ni}$ was chosen; its half life is 98.7 (24) years and the endpoint energy $66.980(15) \mathrm{keV}$. This low energy beta emitter was chosen also because in this energy range the potential energy loss due to escape of Bremsstrahlung photons from the detector can be safely neglected, as has been confirmed by Monte Carlo simulation. This makes the validation of the experimental method more reliable because no correction for this energy loss needs to be applied. The aim of this development remains of course the determination of the shape factors particularly for beta emitters decaying via forbidden transitions, whose spectra are difficult to calculate and often experimentally not well known, and up to endpoint energies around $1 \mathrm{MeV}$. 
For the energy range $\sim 500 \mathrm{keV}$ to several $\mathrm{MeV}$, a new beta spectrometer based on semiconductor detectors is currently being implemented at LNHB (Bisch et al., 2013).

\section{Metallic magnetic calorimeters for beta spectrometry}

Like most cryogenic detectors, metallic magnetic calorimeters (MMCs) are thermal detectors: the energy $E$ of each individual particle is measured as a temperature rise $\Delta T=E / C$ where $C$ denotes the total heat capacity of the detector. Since the specific heat of most materials strongly decreases with temperature, the signal $\Delta T$ is maximized when operating the detector at very low temperature: usually $10-50 \mathrm{mK}$. At the same time, in this temperature range thermodynamic fluctuation noise and other thermal noises are very small, hence a very high signal-to-noise ratio of MMCs is achievable, an essential condition for low threshold and high energy resolution. A thermal link connects the detector to a heat sink kept at constant temperature in order to restore the initial temperature after each particle interaction. The signal decay time $\tau$ is determined by the thermal conductance $G$ of the link: $\tau=C / G$. Decay times of a few hundred $\mu$ s to several ms are typical, allowing for count rates of order $10 \mathrm{~s}^{-1}$.

Mostly MMCs have a metallic particle absorber, often made of gold, and the temperature rise is measured by a metallic paramagnetic thermometer in good thermal contact with the absorber. The name "metallic magnetic calorimeters" refers to the metallic nature of the thermometer that is necessary for a fast response. The thermometer is composed of gold and a small concentration of erbium (of order $0.1 \%$ ), and its magnetization in the presence of a magnetic field (typically a few $\mathrm{mT}$ ) strongly varies with temperature. This variation upon a particle interaction and the 
consecutive temperature rise is measured with a SQUID (Superconducting Quantum Interference Device), a very low noise magnetometer also operating at cryogenic temperatures.

For beta spectrometry, the beta emitter is enclosed inside the absorber: it can either be deposited on a metal foil constituting half of the absorber; it is then covered by a foil forming the second half of the absorber. Or it can be deposited on a separate thin metal foil that is then sandwiched between the two absorber foils. In order to tightly enclose the source, the metal foils are diffusion welded to each other. The minimum absorber thickness necessary to fully stop all beta particles is determined by Monte Carlo simulation. This way, the detection efficiency is virtually $100 \%$ between the detection threshold and the endpoint of the spectrum. The area of the absorber foils depends on the area required to deposit the beta emitter. Mostly $1 \mathrm{~mm}^{2}$ is sufficient.

Care must be taken to exclude all possible sources of distortion of the experimental spectra, like the following:

(a) Detector non-linearity.

The linearity of the MMCs, including electronics and data acquisition system, has been tested and is very good, see $\S 4.1$.

(b) Pile-up

The count rate, i. e. source activity, must be chosen in dependence of the signal decay time in order to keep pile-up at a very small level. It can be further reduced by application of dead time 
and careful pulse shape analysis. Any remaining unresolved pile-up would not be noticed within the spectrum and could cause spectrum distortion, but it would show up beyond the endpoint of the spectrum. In the measurements presented in this paper it is negligible.

(c) Energy loss by escape of Bremsstrahlung photons

This issue has been checked by Monte Carlo simulation. For the low energy beta emitters presented in this paper it is negligible, but for higher energy emitters (maximum energy several hundred $\mathrm{keV}$ ) it must be corrected for.

(d) Atomic excitation

A small part of the beta energy can be expended by excitation of the electrons of the daughter atom: shake-up or shake-off. This effect can distort the spectrum if the source is external to the detector. By enclosing the source in the absorber, this fraction of the beta energy is also detected in the form of heat and integrated in the total measured energy.

\section{(e) Self-absorption}

Partial loss of beta energy in the source is one of the most common causes of spectrum distortion in beta spectrometry. With the source enclosed in the absorber of a MMC, one would expect that this part of energy will also be detected in the form of heat. However our measurements have revealed that this is not always true, see $\S 4.1$. The influence of the source turned out to be the most critical issue in beta spectrometry with MMCs. 


\section{Exchange effect}

The exchange effect is an atomic effect that is mostly not taken into account in the calculation of beta spectra, although for some nuclides it has a stronger influence than the screening effect, the nuclear size effect or the radiative corrections. It corresponds to the case that a beta electron is not directly emitted to the continuum, but created into a bound orbital of the daughter atom, accompanied by the simultaneous emission of a bound electron from the same orbital to the continuum. The exchange effect has an influence on the spectrum shape: it enhances the global beta emission probability, with the enhancement increasing towards low energies.

The first theoretical description of the exchange effect, taking into account only the 1 s electrons, predicted a reduced emission probability at low energy (Bahcall, 1963). A more complete treatment, including higher orbitals, came to the contrary conclusion that the emission probability increases at low energies (Harston and Pyper, 1992). Experimental evidence has been reported only twice up to now (Lowry et al., 1993; Angrave et al., 1998). These measurements were also carried out with cryogenic detectors (of different type than MMCs), but with energy thresholds of several $\mathrm{keV}$ where the influence of the exchange effect is still relatively small. Both of them observed en enhancement of the emission probability at low energies. MMCs can probe the exchange effect down to much lower energies.

The evaluation of the exchange effect has been implemented in the code BetaShape, developed at LNHB, which in its present form calculates the shape of allowed and unique forbidden transitions. Details on the formalism and computational approach for the calculation of the exchange effect are given in Mougeot et al., 2012. 


\section{Measurements of the beta spectrum of ${ }^{63} \mathrm{Ni}$}

\subsection{Dried sources}

Several MMCs were made with ${ }^{63} \mathrm{Ni}$ sources prepared by drying a small drop of $\mathrm{NiCl}_{2}$ solution. The linearity of the detectors was checked using the gamma and X-rays of a combination of ${ }^{55} \mathrm{Fe},{ }^{109} \mathrm{Cd}$ and ${ }^{241} \mathrm{Am}$ sources. A linear fit was applied to the measured positions of $\mathrm{X}$ - and gamma-ray lines as a function of the tabulated line energies. In the energy range $5.9 \mathrm{keV}-$ $88 \mathrm{keV}$ covered by the photon sources the maximum deviation of the measured line positions from the linear fit was $0.1 \%$ and no trend of the deviations with energy was observed. So the detectors can be considered as linear. For the final spectra only the $59.5 \mathrm{keV}$ gamma line of ${ }^{241}$ Am was used for energy calibration.

Fig. 1 shows several of the experimental spectra together with theoretical spectra calculated with and without the exchange effect using the code BetaShape. All experimental spectra differ from one another and from theory. It is clear, however, that the agreement with the theoretical spectrum including the exchange effect is much better than neglecting the exchange effect. The reason for the differences between the experimental spectra is not clear. The most likely explanation appears to be that part of the beta energy deposited in the $\mathrm{NiCl}_{2}$ forming the source goes into the creation of metastable states like electron-hole pairs whose energy is not transformed to heat on a relevant time scale (McCammon et al., 1985), so that not the entire beta energy is measured. 


\subsection{Electroplated sources}

Given the difficulties encountered with dried sources, several ${ }^{63} \mathrm{Ni}$ sources were prepared by electroplating. Some details on the source preparation are given in Le-Bret et al., 2013. The spectrum measured with one of these sources is presented in fig. 2, again together with theoretical spectra calculated with and without exchange effect. The FWHM energy resolution of this detector is $51 \mathrm{eV}$ for the $59.5 \mathrm{keV}$ line and the energy threshold is $200 \mathrm{eV}$. The experimental spectrum confirms very well the theoretical spectrum including the calculation of the exchange effect, whereas theory without exchange effect is clearly discarded. A spectrum measured with another electroplated source, enclosed in an absorber made of silver instead of gold, confirms the shape of the spectrum in fig. 2, although with less statistics and a higher noise level. The enhancement of the emission probability due to the exchange effect goes up to $23 \%$ at the energy threshold $(200 \mathrm{eV})$ while the mean energy of the beta emission is lowered by $2.3 \%$.

\section{The spectrum of ${ }^{241} \mathrm{Pu}$ revisited}

After the strong evidence for the importance of the exchange effect in the case of ${ }^{63} \mathrm{Ni}$ the question arose to what extent the discrepancy between the experimental and theoretical spectra of ${ }^{241} \mathrm{Pu}$ published in Loidl et al., 2010 may be due to the omission of the exchange effect in the calculation of the theoretical spectrum. As mentioned before, the code BetaShape can calculate only allowed and unique forbidden transitions, whereas ${ }^{241} \mathrm{Pu}$ decays via a first forbidden nonunique transition. It can be shown, however, that in the case of ${ }^{241} \mathrm{Pu}$ the approximation by an allowed transition should introduce only very small errors (Mougeot et al., 2012). In fig. 3 the 
theoretical spectrum of ${ }^{241} \mathrm{Pu}$ including the exchange effect has been added to the spectra presented in Loidl et al., 2010. It is evident that the agreement between experimental and theoretical spectra is much better when the exchange effect is included. The remaining discrepancy is most likely due to the use of a dried source.

\section{Conclusion}

The beta spectrum of ${ }^{63} \mathrm{Ni}$ was measured with several metallic magnetic calorimeters and different types of sources. The spectrum of ${ }^{241} \mathrm{Pu}$ had been measured and published previously (Loidl et al., 2010). The accurateness of the spectra measured with metallic magnetic calorimeters depends crucially on the quality of the sources. The use of dried sources may substantially distort the spectra, whereas a metallic source deposit obtained by electroplating proved to provide accurate spectra. For nuclides that cannot be electroplated in metallic form, such as ${ }^{241} \mathrm{Pu}$ for instance, direct implantation of the radioactive nuclei into the absorber material is a possibility to obtain excellent sources.

The experimental spectra were compared with theoretical spectra calculated either considering only the usual corrections for the screening and nuclear size effects and radiative corrections, or taking additionally into account the exchange effect. The measurements clearly confirm the importance of the exchange effect in the low energy part of these spectra. The impact of the exchange effect is much less pronounced for high energy beta emitters or low mass nuclei. But in some cases, in particular those presented in this paper, it must be taken into account if the precise shape of beta spectra is needed. This is necessary for instance for the calculation of the detection efficiency in liquid scintillation counting of pure beta emitters. For the example of ${ }^{63} \mathrm{Ni}$ 
measured by the TDCR method, a relative bias of 0.5 to $1 \%$ due to the omission of the exchange effect has been estimated.

\section{References}

Angrave, L. C., Booth, N. E., Gaitskell, R. J., Salmon G. L., and Harston, M. R., 1998. Measurement of the atomic exchange effect in nuclear $\beta$ decay. Phys. Rev. Lett. 80 (8) $1610-1613$.

Bahcall, J. N., 1963. Overlap and exchange effects in beta decay. Phys. Rev. 129 (6) 2683-2694.

Bisch, C., Mougeot, X., Bé, M.-M. and Nourreddine, A.-M., 2013. Development of a system for measuring the shape of $\beta$ spectra using a semiconductor Si detector. Proc. International Conference on Nuclear Data for Science and Technology, ND 2013, New York, 4-8 March 2013, to be published in Nuclear Data Sheets.

Enss, C. Fleischmann, A., Horst, K., Schönefeld, J., Sollner, J., Adams, J. S., Huang, Y. H., Kim, Y. H. and Seidel, G., 2000. Metallic Magnetic Calorimeters for Particle Detection. J. Low Temp. Phys. 121 (3-4) 137-176.

Fleischmann, A., Enss, C. and Seidel, G., 2005. Metallic Magnetic Calorimeters, in Cryogenic Particle Detectors. Topics Appl. Phys. 99, Enss, C. (Ed.), Springer Berlin/Heidelberg, $151-216$.

Harston, M. R. and Pyper, N. C., 1992. Exchange effects in $\beta$ decays of many-electron atoms. Phys. Rev. A 45 (9) 6282-6295.

Le-Bret, C., Mougeot, X., Loidl, M., Cassette, P. and Rodrigues, M., 2013. Evidence for exchange effect in the $\beta$ spectrum of ${ }^{63} \mathrm{Ni}$ measured with cryogenic detectors. Submitted to Phys. Lett. B. 
Loidl, M., Rodrigues, M., Censier, B., Kowalski, S., Mougeot, X., Cassette, P., Branger, T., and Lacour, D., 2010. First measurement of the beta spectrum of ${ }^{241} \mathrm{Pu}$ with a cryogenic detector. Appl. Rad. Isot. 68 (7-8) 1454-1458.

Lowry, M. M., Deptuck, D. and Girit, I. C., 1993. Beta spectrum studies with cryogenic microcalorimeters. J. Low Temp. Phys. 93 (3-4) 239-244.

McCammon, D., Juda, M., Zhang, J., Kelley, R. L., Moseley, S. H. and Szymkowiak, A. E., 1986. Thermal detectors for high resolution spectroscopy. IEEE Trans. Nucl. Sci. 33, 236-239.

Mougeot, X., Bé, M.-M., Bisch, C. and Loidl, M., 2012. Evidence for the exchange effect in the $\beta$ decay of ${ }^{241} \mathrm{Pu}$. Phys. Rev. A 86, 042506-1 - 042506-7. 


\section{Figure captions:}

Figure 1: Spectra of dried ${ }^{63} \mathrm{Ni}$ sources measured with various MMCs compared with theoretical spectra with and without exchange effect. The experimental spectra are normalized with respect to the theoretical spectrum including the exchange effect between 0 and $55 \mathrm{keV}$.

Figure 2: Spectrum of an electroplated ${ }^{63} \mathrm{Ni}$ source measured with a MMC ("rugged" black line), compared with theoretical spectra with (smooth solid line) and without (dashed line) exchange effect.

Figure 3: Spectrum of ${ }^{241} \mathrm{Pu}$ measured with a MMC and presented in Loidl et al., 2010, compared with theoretical spectra with and without exchange effect. 


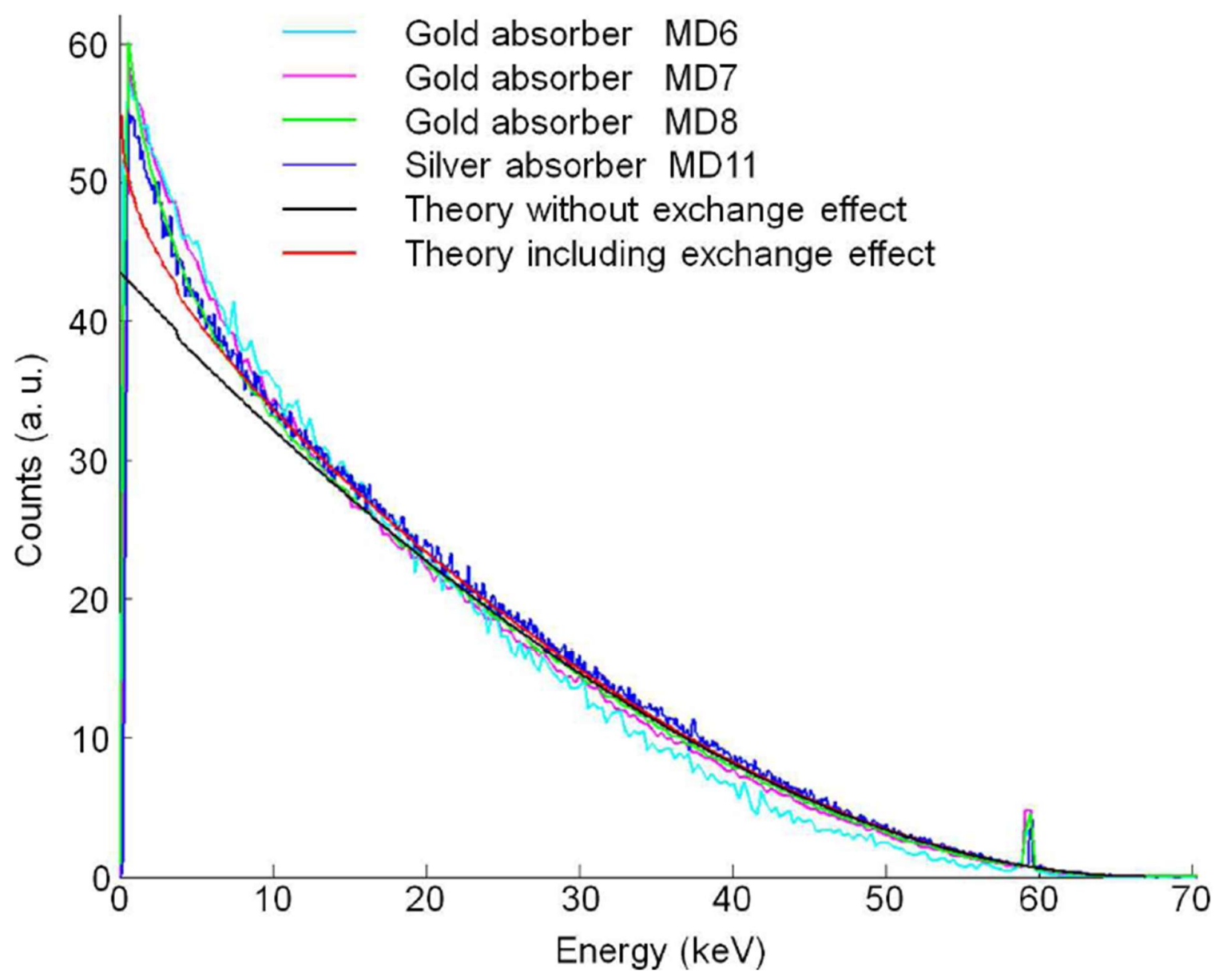

Figure 1 


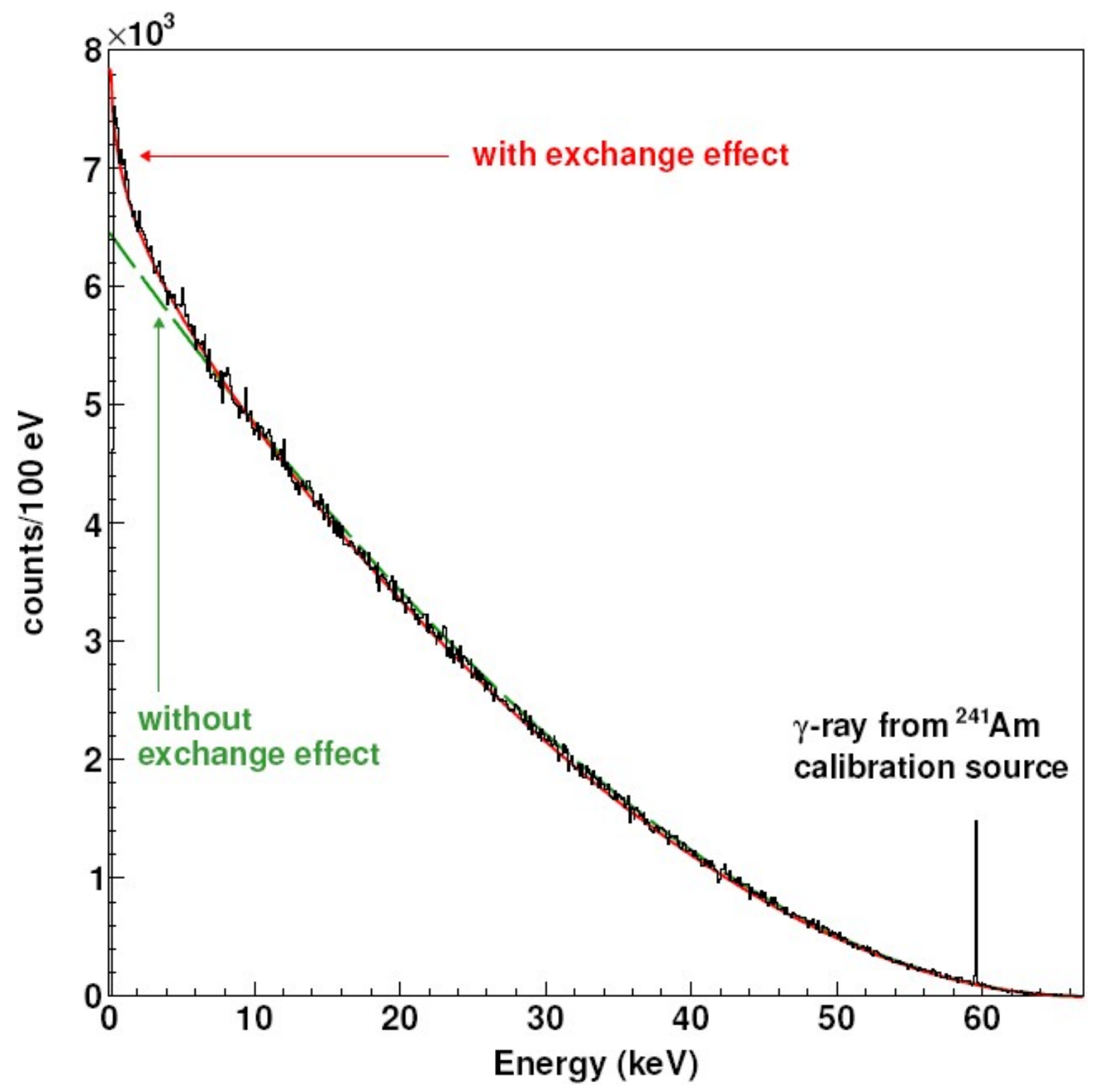

Figure 2 


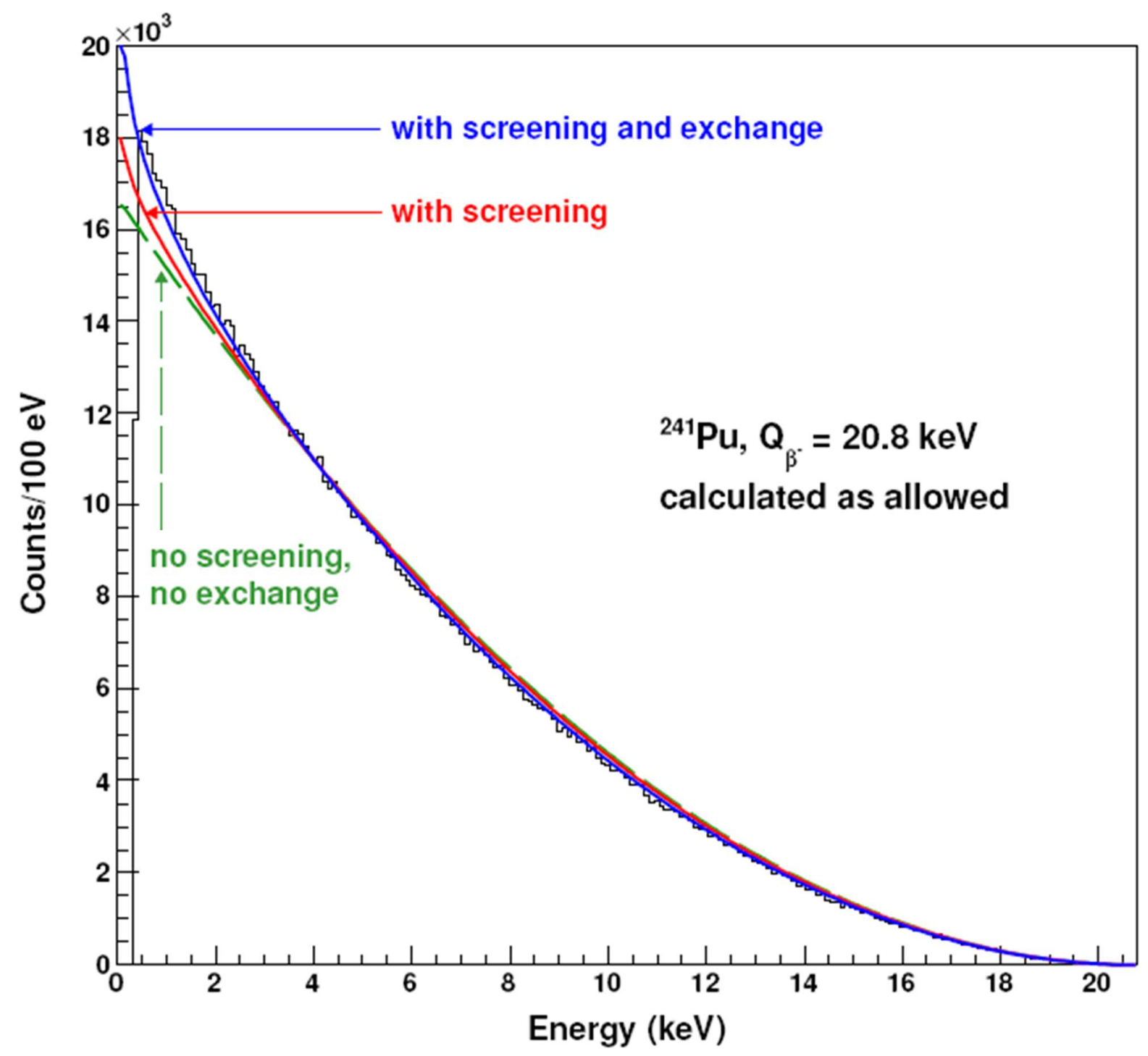

Figure 3 